\title{
Exploration of Human Ageing by 3D-Laser Scanning Anthropometry
}

\author{
Henry LÖFFLER-WIRTH ${ }^{\star 1,2}$, Alexander FRENZEL ${ }^{1}$, Markus LÖFFLER ${ }^{1,2,3}$, Hans BINDER ${ }^{1,2}$ \\ ${ }^{1}$ Interdisciplinary Centre for Bioinformatics, Leipzig University, Germany; \\ ${ }^{2}$ LIFE, Leipzig Research Center for Civilization Diseases, Leipzig University, Germany; \\ ${ }^{3}$ Institute for Medical Informatics, Statistics and Epidemiology, Leipzig University, Germany \\ DOI: $10.15221 / 17.026 \quad$ http://dx.doi.org/10.15221/17.026
}

\begin{abstract}
Three-dimensional whole body scanning is an emerging technology for anthropometric assessment in epidemiological studies. We recently analysed 3D whole body scanning data of nearly 10,000 participants collected from the adult population of Leipzig. We were able to aggregate the body measures provided by the scanner into meta-measures, each representing one relevant dimension of the body shape, and to identify distinguishable clusters of body shapes. In the next step, we now study specifics of the so-called body types upon ageing to understand how meta-measures change during ageing. Further we investigate the distribution of participants in the body types with regard to stratification by age and gender. It becomes clear, that age significantly influences body shape, embodied by characteristic changes of the meta-measures representing systematic shifts in body proportions upon ageing, and by a marked developmental trajectory of body typing.
\end{abstract}

Keywords: $3 d$ body scanning, anthropometry, physiological development, ageing

\section{Introduction}

Three-dimensional (3D-) body scanning offers accurate, reproducible and cheap anthropometric characterization of whole persons' body shapes in few seconds of measurement. Beside widespread commercial applications like apparel design and ergonomics [1,2], body scanning is increasingly utilized in medial context, e.g. for cosmetic and reconstructive surgery [3,4], and recently in medical and health research to study anthropometry of hundreds to thousands of participants in large cohort studies with high accuracy $[5,6]$. These new and amended data are expected to improve the diagnostics of many diseases, however, current applications usually don't consider the whole information provided by the set of body measures and, instead, use only a small part of them to estimate simple body indices such as body mass index (BMI) or waist-to-hip circumference ratio (WtH) [7-10].

3D-body scans were applied in the Leipzig Research Center for Civilization Diseases (LIFE), conducting the largest population based study with an extensive phenotyping of urban individuals in Germany to date [11]. Examination of 10,000 randomly selected adult participants from Leipzig (LIFE-ADULT) was performed to investigate prevalences, early onset markers, and the role of lifestyle factors of major civilization diseases. LIFE-ADULT covers an age range from 40-80 years.

Previously, we developed an analysis pipeline for 3D body scanning data and applied this software to the LIFE-ADULT data [12]. There we systematically analysed the complete set of body measures provided by 3D-body scanning. It however remains unclear how body shapes change upon ageing, and how body types relate to this physiological development. These questions will be addressed in this publication by a systematics view on the body types as a function of age.

\section{Data and Methods}

\subsection{D body scanning and data preparation}

We analyzed anthropometric 3D body scanner data collected in the LIFE-ADULT study between 2011 and 2014 [11]. 3D body surface scanning was performed by a 'Vitus Smart XXL' 3D laser scanner (Human Solutions GmbH, Kaiserslautern, Germany) which provides an image of the body surface of each participant. In total 155 body measures were extracted from this image using AnthroScan 2.9.9 software. Measurement and data generation are in agreement with ISO 20685, the international standard for 3-D scanning methodologies for internationally compatible anthropometric databases.

We considered 134 body measures of 8,499 participants including length and girth measures, weight, and the indices 'body mass index' (BMI [13]), 'waist to hip circumference ratio' (WtH [14]), 'waist circumference to height ratio' (WHtR [15]) and 'a body shape index' (ABSI [16]).

\subsection{Clustering provides meta-measures and body types}

We recently developed an analysis pipeline for 3D body scanning data and applied this software to the LIFE-ADULT data [12]. In brief, we applied self-organizing map (SOM) machine learning to aggregate body measures provided by the scanner software into a roughly tenfold reduced set of meta-measures, 
each representing a cluster of correlated single body measures. In result, body shape of each participant is anthropometrically characterized by a set of 13 meta-measures, each of which estimates one relevant dimension of body shape. An overview of the meta-measures and their localizations are given in Figure 2a. In a second clustering step, participants were aggregated into 15 body types (see [12] for detailed discussion): We found two body types which collect larger fractions of both male and female participants (B1 and B2), representing body shapes which lack of gender specifics. Six body types collect virtually exclusively women ( $F 1-F 6)$, while seven body types are male specific (M1-M7). The body types not only differ in the corresponding body shapes, but also in age, body height, weight and BMI characteristics.

\section{Results and discussion}

\subsection{Ageing as seen by classical body indices}

We stratified the 8,499 participants by sex and age to evaluate development of classical anthropometrical characteristics upon ageing. Particularly, we generated boxplots of body height, weight, body mass index (BMI) and waist-to-hip circumference ratio $(\mathrm{WtH})$ arranged in 5-years intervals (Figure 1): At all ages, men are - on average - taller than women as expected (Figure 1a). Body heights of both sexes start to decrease from an age of about 50 years. The lower quantile of body height in younger ( $<50$ years) participants approximately corresponds to the upper quantile in older ones $(>70$ years).

Weight of the participants alters in an opposed fashion compared with participant's height, i.e., it increases with age up to the 55-59 years interval, and then declines (Figure 1b). Combination of these characteristic courses of height and weight result in increasing BMI values up to about 60 years, and then BMI levels off. BMI curves of both sexes are very similar, however men show a slightly higher mean BMl than women. Note that from an age of about 60 years, more than $25 \%$ of participants are obese $(\mathrm{BMI} \geq 30)$.

Age-dependency of WtH resembles that of BMI, where however men reveal markedly higher values compared to women. Among people older than 50 years, more than $50 \%$ show an 'apple-like' body shape ( $\mathrm{WtH} \geq 0.8$ and 0.9 in women and men, respectively), which is found to associate with higher health risk [14].

Importantly, the body indices remain, on average, virtually invariant for women and men older than 60 years which makes them inappropriate to discriminate age-dependent trends for elderly people, and therefore not suitable to describe development upon ageing.

(a)

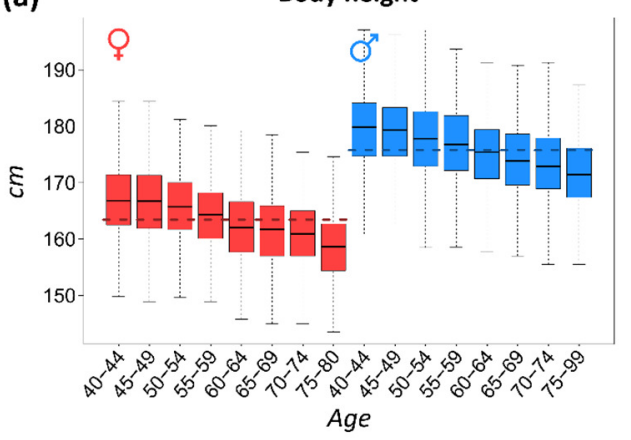

(c)

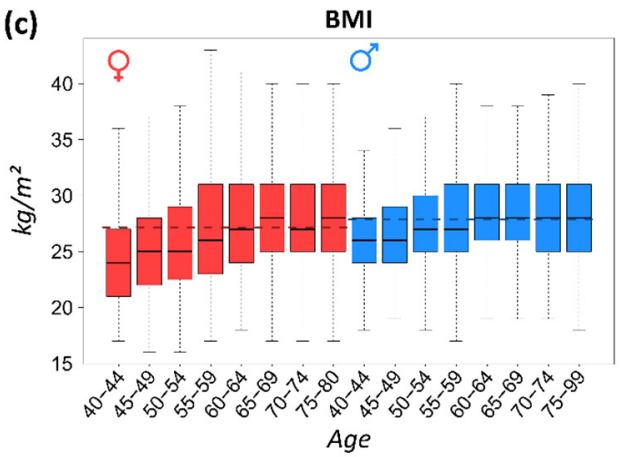

(b)

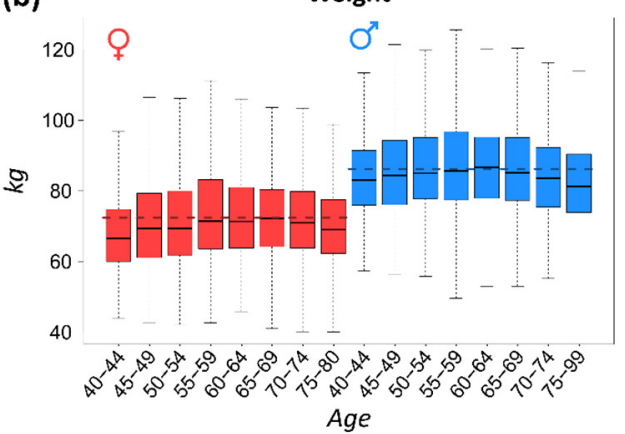

(d)

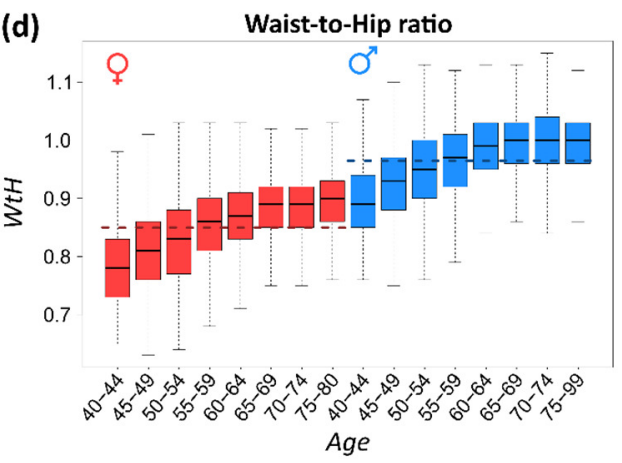

Figure 1: Classical anthropometric measures characteristically change upon ageing. (a) Body height, (b) weight, (c) BMI and (d) waist-to-hip circumference ratio are given as boxplots stratified by sex and age, respectively. Dashed horizontal lines refer to mean values in women and men, respectively. 


\subsection{Meta-measures specifically change upon ageing}

Next we analysed the previously defined body meta-measures for age driven alterations. This set of 13 meta-measures virtually distributes over the whole body (Figure 2a) and therefore comprehensively describes the body shape of a participant [12]. For visualization, we use a polar diagram termed 'bodygram', where each axis refers to one meta-measure (Figure 2b). We generated mean genderspecific bodygrams averaged over all women and men, respectively (Figure 2c, left part). They reveal characteristic differences between women and men such as larger dimensions of the upper body as estimated by the meta-measures $\mathrm{H}-\mathrm{M}$ in men, and larger girth and length dimensions of the legs (meta-measures $\mathrm{C}$ and $\mathrm{F}$ ) in women. The latter result means that the respective leg measures were larger in women in relation to their body height because we used body measures that were normalized with respect to the body height. For an age dependent view, we generated bodygrams after stratification of the participants by decadal age intervals (Figure 2c, right part), and then we calculated differential bodygrams between the 40-49 years and 70-80 years intervals to visualize alterations of the metameasures (Figure 2c, left part). We find meta-measures which change in both sexes similarly, thus reflecting general, i.e. sex-independent age related trends ( $B, K \& M$, and, to less extend, $A, D, E \& H)$. All these meta-measures increase upon ageing except for meta-measure $\mathrm{H}$, which refers to upper body lengths. This indicates a shift of proportions towards smaller torso in relation to body size in older participants. In contrast, increasing meta-measures collect mainly girth measures, which indicates the gain of body volume and consequently of the weight as discussed above. Other meta-measures change in gender-specific fashion: $J$ and $L$ (neck length and arm girths, respectively) markedly increase in women. On the other hand, $\mathrm{C}$ (thick girth) specifically decreases in men, which can be interpreted as a relative redistribution of body mass from legs towards torso, or, in other words, the shift from pear-like to apple-like body shapes.

(a)

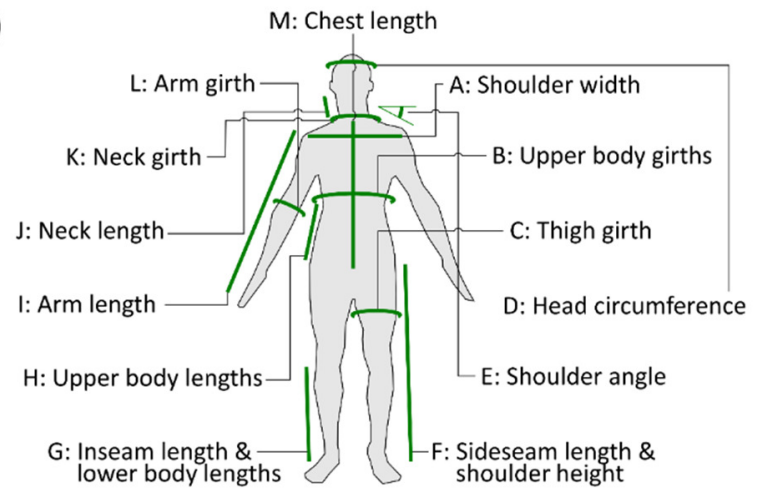

(c)
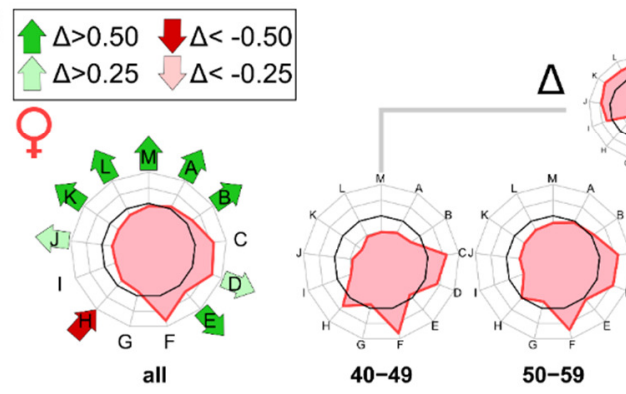

$\Delta$
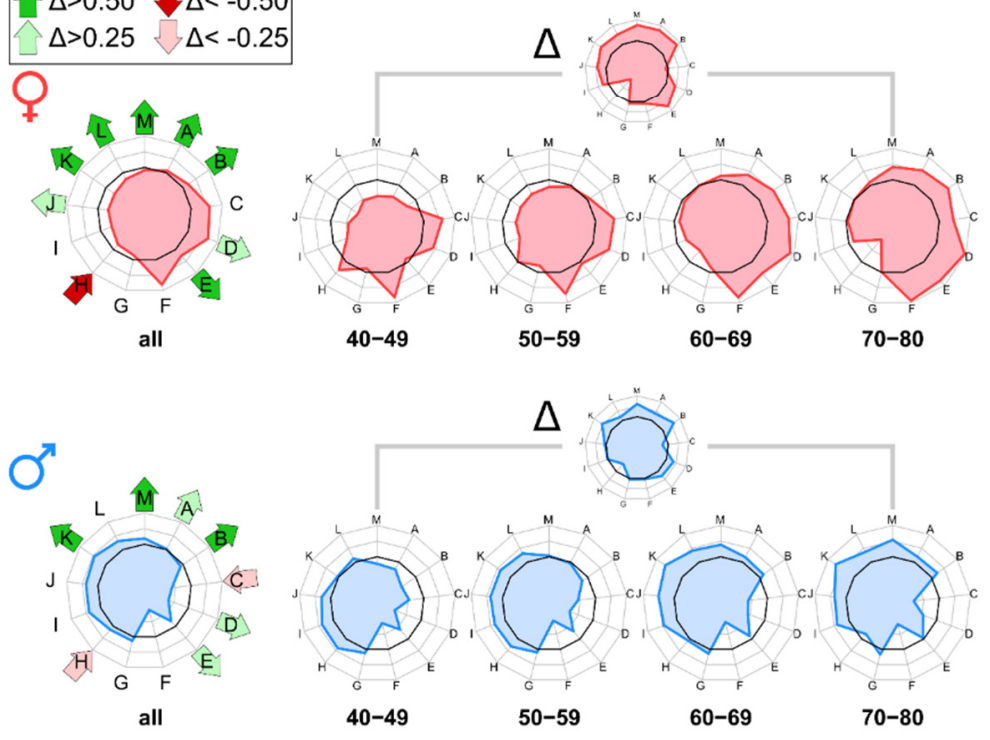

(b) Bodygram:

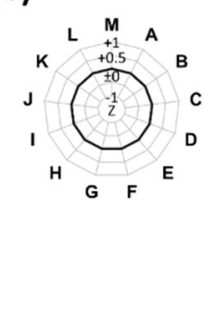

70-80

Figure 2: Body meta-measures reveal common trends with few gender specifics. (a) Localization of the 13 metameasures. (b) Bodygrams are polar diagrams with the meta-measures in Z-units as axes. The black polygon refers to $Z=0$. (c) Bodygrams are shown for male and female participants separately (left part: all ages, right part: stratified by 10-years intervals). Difference bodygrams represent changes of meta-measures from 40-49 to 70-80 intervals, respectively. Meta-measures with marked increase or decrease $(\Delta Z> \pm 0.25)$ are highlighted by arrows. 


\subsection{Ageing body types}

In our previous analysis of the LIFE body scanner data we defined 15 body types in terms of clusters of participants with similar body shape [12]. This directly raises the question, if age-related changes of the meta-measures differs between these body types. Therefore we evaluated bodygrams for female and male body types in 10-years intervals (data not shown): Meta-measures can generally be divided into those that mainly increase, decrease, and almost invariant ones. Increasing courses are found for shoulder angle (E), meaning more hanging shoulder in age, and for chest (I) and arm lengths (M). The latter trends represent an increase of the relative length of the upper body, which, in turn, implies the relative decrease of the dimensions of the lower part of the body. This can be explicitly observed in men in terms of decreasing thigh girths (C). Hence, ageing body types are characterized by the shift of body proportions towards the upper part with relatively short and lean legs.

It also becomes clear that many body types maintain or even emphasize their most prominent characteristics in older age. E.g., body types with tall and lean shape (B1, F1 \& M2) become longer and/or leaner (longer chest \& upper body). Men with broad neck (M4 \& M7) keep this property, and participants with massive upper body (F3 \& M5) additionally become leaner legs. This indicates that, slim body shapes remain slim while obese body shapes remain obese upon ageing. Slim body shapes moreover tend to become even more lean and fragile, whereas obese types stay in the obese state.

We further analysed distribution of participants over the body types as function of age (data not shown). It becomes clear, that the percentage of participants collected into the body types markedly alters with age, and that frequencies of most body types, especially for younger and older people, strongly deviate from the corresponding mean incidence and varies upon ageing.

Interestingly, the frequency distribution changes more strongly for women then for men. These changes in female body typing cover the complete age range of female participants of the LIFE study. Men, on the other hand, change their body shape mainly up to an age of 60 years, which indicates main ageing effects in the context of body shape seem to end at this age.

\section{Summary and conclusion}

We present a novel approach to characterize anthropometrical development upon ageing by utilizing $3 d$-body scanner data. We found age-variant and almost age-invariant features as seen by the metameasures, and we found body types with gender-specific changes. As a general trend we found that body types converge towards older-age specific ones, whereby changes in women are more pronounced and persist in older age than in men. In general, ageing leads to a (relative) shrinking of the upper parts of the torso compared to that of the extremities with the trend for the (relative) increase of leg girths for women and that of arms for men. In summary, we have shown that our systematic scheme captures the diversity of the human body shape and their changes upon ageing with high resolution.

\section{Acknowledgements}

This publication is supported by LIFE - Leipzig Research Center for Civilization Diseases, Leipzig University. This project was funded by means of the European Social Fund and the Free State of Saxony. We thank all participants who were willing to take part in the study and the entire study team for their commitment.

\section{References}

[1] A. Kus, E. Unver, and A. Taylor, "A comparative study of 3D scanning in engineering, product and transport design and fashion design education," Comput. Appl. Eng. Educ., vol. 17, pp. 263-271, 2009.

[2] K. Simmons and C. Istook, "Body measurement techniques: Comparing 3D body-scanning and anthropometric methods for apparel applications," J. Fash. Mark. Manag., vol. 7, no. 3, pp. 306-332, 2003.

[3] K. Koban and R. Giunta, "Using Mobile 3D Scanning Systems for Objective Evaluation of Form, Volume, and Symmetry in Plastic Surgery: Intraoperative Scanning and Lymphedema Assessment," in Proceedings of the 7th International Conference on 3D Body Scanning Technologies, 2016.

[4] J. Rosicky, A. Grygar, P. Chapcak, T. Bouma, and J. Rosicky, "Application of 3D Scanning in Prosthetic \& Orthotic Clinical Practice," in Proceedings of the 7th International Conference on 3D Body Scanning Technologies, 2016.

[5] A. Kuehnapfel, P. Ahnert, M. Loeffler, A. Broda, and M. Scholz, "Reliability of 3D laser-based anthropometry and comparison with classical anthropometry," Sci. Reports, no. May, pp. 1-11, 2016. 
[6] F. Glock et al., "Validity and intra-observer reliability of three-dimensional scanning compared to conventional anthropometry for children and adolescents from a population-based cohort study," Pediatr. Res., Jan. 2017.

[7] P. Treleaven and J. Wells, "3D body scanning and healthcare applications," Computer (Long. Beach. Calif)., vol. 40, no. 7, pp. 28-34, 2007.

[8] J. C. K. Wells, A. Ruto, and P. Treleaven, "Whole-body three-dimensional photonic scanning: a new technique for obesity research and clinical practice.," Int. J. Obes. (Lond)., vol. 32, no. 2, pp. 232-8, Feb. 2008.

[9] L. Jaeschke, A. Steinbrecher, and T. Pischon, "Measurement of Waist and Hip Circumference with a Body Surface Scanner: Feasibility, Validity, Reliability, and Correlations with Markers of the Metabolic Syndrome," PLoS One, vol. 10, no. 3, p. e0119430, Mar. 2015.

[10] J. D. Lin, W. K. Chiou, H. F. Weng, J. T. Fang, and T. H. Liu, "Application of three-dimensional body scanner: observation of prevalence of metabolic syndrome.," Clin. Nutr., vol. 23, no. 6, pp. 1313-23, Dec. 2004.

[11] M. Loeffler et al., "The LIFE-Adult-Study: objectives and design of a population-based cohort study with 10,000 deeply phenotyped adults in Germany," BMC Public Health, vol. 15, no. 1, p. 691, Jan. 2015.

[12] H. Löffler-Wirth et al., "Novel Anthropometry Based on 3D-Bodyscans Applied to a Large Population Based Cohort.," PLoS One, vol. 11, no. 7, p. e0159887, 2016.

[13] A. Keys, F. Fidanza, M. J. Karvonen, N. Kimura, and H. L. Taylor, "Indices of relative weight and obesity," J. Chronic Dis., vol. 25, no. 6, pp. 329-43, 1972.

[14] G. M. Price, R. Uauy, E. Breeze, C. J. Bulpitt, and A. E. Fletcher, "Weight, shape, and mortality risk in older persons: elevated waist-hip ratio, not high body mass index, is associated with a greater risk of death.," Am. J. Clin. Nutr., vol. 84, no. 2, pp. 449-60, Aug. 2006.

[15] M. Ashwell, P. Gunn, and S. Gibson, "Waist-to-height ratio is a better screening tool than waist circumference and BMI for adult cardiometabolic risk factors: systematic review and meta-analysis.," Obes. Rev., vol. 13, no. 3, pp. 275-86, Mar. 2012.

[16] N. Y. Krakauer and J. C. Krakauer, "A new body shape index predicts mortality hazard independently of body mass index.," PLoS One, vol. 7, no. 7, p. e39504, Jan. 2012. 